\title{
A mechanism for achieving zero-lag long-range synchronization of neural activity Gordon Pipa ${ }^{1,2,3}$, Raul Vicente*1,2, Leonardo Gollo ${ }^{4}$, Claudio Mirasso 4 and Ingo Fischer ${ }^{5}$
}

\author{
Address: ${ }^{1}$ Department of Neurophysiology, Max-Planck Institute for Brain Research, Frankfurt, Germany, ${ }^{2}$ Frankfurt Institute for Advanced Studies \\ (FIAS), Frankfurt, Germany, ${ }^{3}$ Massachusetts Institute of Technology (MIT) and Massachusetts General Hospital (MGH), USA, ${ }^{4}$ Universitat de les \\ Illes Balears \& IFISC, Palma de Mallorca, Spain, and ${ }^{5}$ Heriot Watt University, Edinburgh, UK \\ Email: Raul Vicente* - vicente@fias.uni-frankfurt.de \\ * Corresponding author
}

from Eighteenth Annual Computational Neuroscience Meeting: CNS*2009

Berlin, Germany. 18-23 July 2009

Published: 13 July 2009

BMC Neuroscience 2009, I0(SuppI I):P240 doi:I0.II86/I47I-2202-I0-SI-P240

This abstract is available from: http://www.biomedcentral.com/I47I-2202/I0/SI/P240

(c) 2009 Pipa et al; licensee BioMed Central Ltd.

How can two distant neural assemblies synchronize their firings at zero-lag even in the presence of non-negligible delays in the transfer of information between them? Neural synchronization stands today as one of the most promising mechanisms to counterbalance the huge anatomical and functional specialization of the different brain areas. However, and albeit more evidence is being accumulated in favor of its functional role as a binding mechanism of distributed neural responses, the physical and anatomical substrate for such a dynamic and precise synchrony, especially zero-lag even in the presence of non-negligible delays, remains unclear.

Here we propose a simple network motif that naturally accounts for zero-lag synchronization of spiking assemblies of neurons for a wide range of temporal delays. We demonstrate that when two distant neural assemblies do not interact directly but relaying their dynamics via a third mediating single neuron or population and eventually achieve zero-lag coherent firing. Extensive numerical simulations of populations of Hodgkin-Huxley neurons interacting in such a network are analyzed. The results show that even with axonal delays as large as $15 \mathrm{~ms}$ the distant neural populations can synchronize their firings at zero-lag in a millisecond precision after the exchange of a few spikes. The role of noise and a distribution of axonal delays in the synchronized dynamics of the neural popu- lations are also studied confirming the robustness of this sync mechanism. The proposed network module is densely embedded within the complex functional architecture of the brain and especially within the reciprocal thalamocortical interactions where the role of indirect pathways mimicking direct cortico-cortical fibers has been already suggested to facilitate trans-areal cortical communication.

In summary the robust neural synchronization mechanism presented here arises as a consequence of the relay and redistribution of the dynamics performed by a mediating neuronal population. In opposition to previous works, neither inhibitory, gap junctions, nor complex networks need to be invoked to provide a stable mechanism of zero-phase correlated activity of neural populations in the presence of large conduction delays.

\section{Acknowledgements}

This work was partially supported by the Hertie Stiftung and the EC Project GABA (contract 043309).

\section{References}

I. Fischer I, Vicente R, Buldu JM, Peil M, Mirasso CR, Torrent MC, Garcia-Ojalvo J: Zero-lag long-range synchronization via dynamical relaying. Phys Rev Lett 2006, 97:123902.

2. Vicente R, Gollo LL, Mirasso CR, Fischer I, Pipa G: Dynamical relaying can yield zero time lag neuronal synchrony despite long conduction delays. PNAS 2008, 105:17157-17162. 